\title{
JET ENGINE INLET DISTORTION SCREEN AND DESCRIPTOR EVALUATION
}

\author{
Jiri Pecinka ${ }^{a, *}$, Gabriel T. Bugajski $^{b},{\text { Petr } \mathrm{KMOCH}^{a} \text {, Adolf Jilek }}^{a}$ \\ a Department of Airforce and Aircraft Technology, University of Defence, Brno, Czech Republic \\ ${ }^{b}$ Air Force Operational Test and Evaluation Center Detachment 5, Operating Location Czech Republic, Brno, \\ Czech Republic \\ * corresponding author: jiri.pecinka@unob.cz
}

Abstract. Total pressure distortion is one of the three basic flow distortions (total pressure, total temperature and swirl distortion) that might appear at the inlet of a gas turbine engine (GTE) during operation. Different numerical parameters are used for assessing the total pressure distortion intensity and extent. These summary descriptors are based on the distribution of total pressure in the aerodynamic interface plane. There are two descriptors largely spread around the world, however, three or four others are still in use and can be found in current references.

The staff at the University of Defence decided to compare the most common descriptors using basic flow distortion patterns in order to select the most appropriate descriptor for future department research. The most common descriptors were identified based on their prevalence in widely accessible publications. The construction and use of these descriptors are reviewed in the paper. Subsequently, they are applied to radial, angular, and combined distortion patterns of different intensities and with varied mass flow rates.

The tests were performed on a specially designed test bench using an electrically driven standalone industrial centrifugal compressor, sucking air through the inlet of a TJ100 small turbojet engine. Distortion screens were placed into the inlet channel to create the desired total pressure distortions. Of the three basic distortions, only the total pressure distortion descriptors were evaluated. However, both total and static pressures were collected using a multi probe rotational measurement system.

KEYWORDS: pressure inlet distortion; distortion screen; distortion coefficient; small jet engine.

\section{INTRODUCTION}

This paper is focused on total pressure inlet distortions. The total pressure that drops in regions with flow separation or turbulence correlates well with the distribution of velocities at the compressor inlet which defines angles of attack to the compressor blades and thus influences the compressor performance and operability. Accordingly the pressure distribution at the Aerodynamic Interface Plane (AIP) defines the change of the surge margin.

The total pressure distortions may appear during aircraft operations as a result of an S-shaped inlet duct [1-4], flow separation or wake initiated by a high angle of attack or by a shock wave. A variety of methods can be used for creation of such distortions for compressor and engine rig testing. For generating of steady-state total-pressure, distortion wire-mesh screens are often implemented [5]. These relatively simple-to-fabricate and easy-to-use distortion screens were selected for analysis at the University of Defence.

Measurements of total pressures at the AIP of the industry minimum of 40 points distributed within the plane, results in a very complex pressure distortion map (e.g. Fig. 6). This type of distortion map provides some insight into the distortion by itself, but it is difficult to compare many different conditions in this manner. As a result, quantitative measures, commonly known as distortion descriptors or distortion parameters, are used to describe the distortion.

Many descriptors were developed by different engine manufacturers in the $60^{\prime} \mathrm{s}$ and $70^{\prime} \mathrm{s}$. There have been some efforts to catalogue and compare different descriptors throughout the years [6] 8 . The descriptors differ in complexity. Some of them summarize the pressure distribution into a single number, other descriptors divide the AIP into several areas and describe the pressure difference among them in circumferential and radial direction as well as intensity and extent using different descriptor elements. Methods that correlate the distortion descriptors with change of the surge margin are also different; however, there does not seem to be a unique form of utilization of the descriptors to correlate with the surge margin reduction that will meet the accuracy requirements for every compressor. Independently of the method used, its accuracy and implementation laboriousness will depend on the distortion descriptor used and the level of its correlation with the particular compressor properties.

In modern times, the field of commonly used descriptors have winnowed primarily down to two; one in the US (SAE 1420) and one in the UK and Europe (DC60) [2, although others can still be found in cur- 


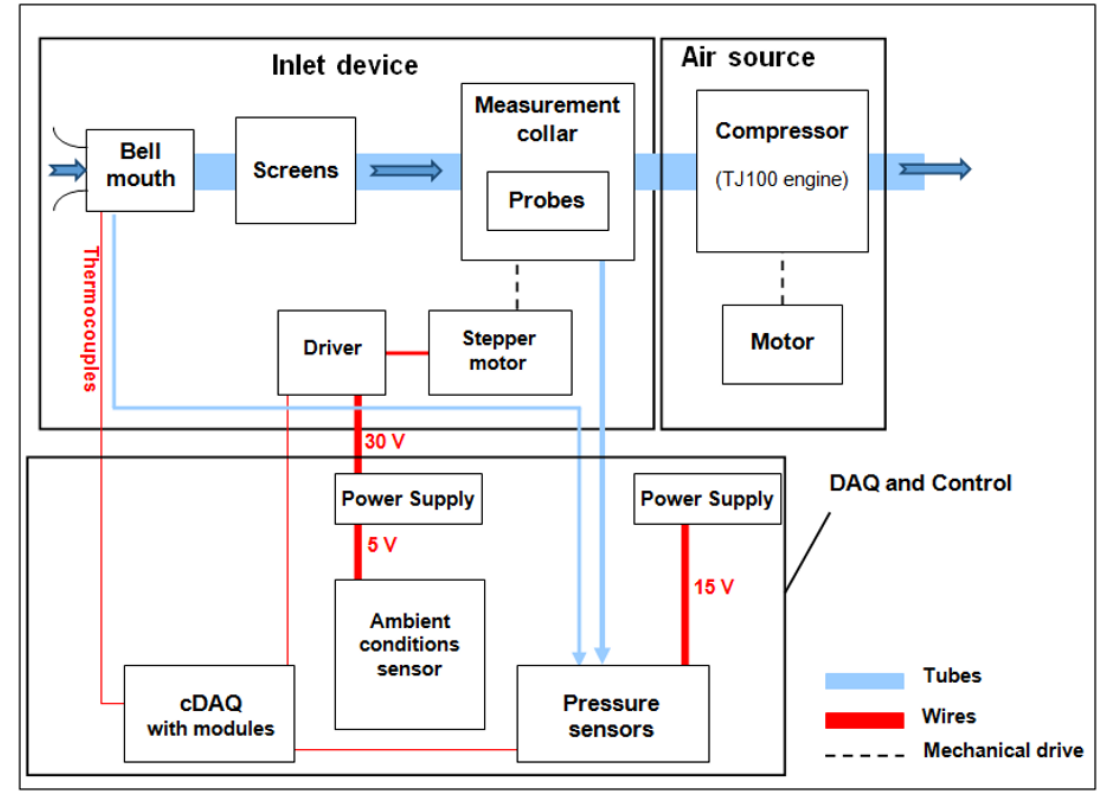

Figure 1. Test Setup Scheme.

rent references. Despite extensive documentation to other areas, the influence of flow velocity, distortion screen size and density to the generated distortion was not found in the literature.

Accordingly, it was decided to evaluate the most commonly used distortion descriptors for basic types of total pressure distortions created by several screens. The first goal was to reveal (confirm) the dependency of the descriptors separately on the change of the total pressure in the circumferential and radial direction, level (intensity) of the pressure drop and with the change of air mass flow rate. The second was to confirm the suitability of the proposed distortion screen designs and gain a basic knowledge about the levels of distortions that can be generated through their implementation. The tests presented in this paper were performed on a full scale model system of the TJ100 small jet engine inlet. The system is briefly described later in this paper, while an overview of the full system is provided in Fig. 1. In order to decrease the costs of the initial phase of the tests, the inlet was connected to a stand-alone industrial compressor instead of the TJ100 engine. The test methodology was refined and descriptor comparison tests were performed based on this setup. The most important results and their analysis are presented here. Following the testing, the same device will be used with actual TJ100 engine to evaluate the influence of distortion generated by selected screens on the engine performance and surge margin change.

\section{Distortion Descriptors}

There are a variety of different numerical descriptors of total pressure distribution available in different references. However, their usage differs from author to author and company to company. Sometimes, the same descriptor is used with minor differences or poorly described. Definitions of descriptors chosen for comparative analysis based on research that the authors of this paper completed are presented here.

\subsection{SAE AIR1419 STANDARD DESCRIPTOR}

The SAE standard descriptor is the most complex descriptor widely used. The descriptor elements separately define the intensity in circumferential and angular directions as well as angular distortion extent and the number of low pressure regions in the circumference. Moreover, all descriptor elements are defined separately for all of the probe rings in the AIP. The following characteristics are based on [5, 9].

Circumferential distortion intensity (CDI). The circumferential distortion intensity element $(\triangle P C / P)$ is a numerical indication of the magnitude of total pressure defect for ring $i$ :

$$
C D I=\left(\frac{\Delta P C}{P}\right)_{i}=\frac{\left(P_{\mathrm{R}, \mathrm{AV}}\right)_{i}-\left(P_{\mathrm{L}, \mathrm{AV}}\right)_{i}}{\left(P_{\mathrm{R}, \mathrm{AV}}\right)_{i}} .
$$

Extent. The circumferential distortion extent element $\left(\theta^{-}\right)_{i}$, is the angular region in degrees in which the pressure is below the ring $i$ average total pressure. It is defined by the intersection between the ring average pressure and the linear interpolation, which subtends the low-pressure region $\left(\theta_{1_{i}}, \theta_{2_{i}}\right)$ :

$$
\left(\theta^{-}\right)_{i}=\theta_{2_{i}}-\theta_{1_{i}} .
$$

Multiple per revolution $(M P R)$. The circumferential distortion element $(M P R)_{i}$ is a numerical indication of the "effective" number of low-pressure regions for ring $i$ :

$$
(M P R)_{i}=\frac{\sum_{k=1}^{Q}\left(\frac{\Delta P C}{P}\right)_{i k} \theta_{i k}^{-}}{\max \left[\left(\frac{\Delta P C}{P}\right)_{i k} \theta_{i k}^{-}\right]} .
$$


Radial Distortion Intensity $(R D I)$. The radial distortion intensity element $\left(\Delta(P R / P)_{i}\right.$ is the difference between the face-average pressure and the ring-average pressure relativized to the face-average pressure for ring $i$ :

$$
R D I=\left(\frac{\Delta P R}{P}\right)_{i}=\frac{P_{\mathrm{F}, \mathrm{AV}}-\left(P_{\mathrm{R}, \mathrm{AV}}\right)_{i}}{P_{\mathrm{F}, \mathrm{AV}}} .
$$

\subsection{Distortion COEFFicient DC60 (90)}

The DC60 distortion parameter is widely used. It has been derived by Rolls Royce and is described in many papers and text books e.g. [2, 10]. It has been used extensively in the European fighter programs Tornado and Eurofighter [8. It is also built into the calculations of compressor performance in the GasTurb program [11. The DC60 coefficient is employed to quantify the degree of inlet pressure distortion in a fixed angular region. Typically, the angular region is $60^{\circ}$ and hence termed the $D C 60$, although according to [10], $90^{\circ}$ is often considered for modern compressors. The coefficient is defined as the difference between the average total pressure in the most distorted $60^{\circ}$ sector and the mean total pressure for the full $360^{\circ}$ of the AIP (face average pressure), divided by the average inlet dynamic pressure. It is limited to one-per-revolution, circumferential total pressure non-uniformities:

$$
D C 60=\frac{P_{\mathrm{L} 60}-P_{\mathrm{F} . \mathrm{AV}}}{P_{\mathrm{DI}}} .
$$

Some references [8] define the reference dynamic pressure at the AIP instead of the inlet. This difference effects the resultant calculation, because the dynamic pressure value at the AIP is influenced by the pressure distortion and thus gives similar but slightly smaller DC60 values. For the purposes of this paper, the inlet dynamic pressure (i.e., in the undistorted plane) was used.

\subsection{Distortion index $(D I)$}

The distortion index is defined as the difference between maximum and minimum total pressures relativized to the face average pressure in the AIP. It does not take into account the size of the low pressure region or the fact that there may be several low pressure regions. This parameter does not distinguish between circumferential and radial variations of the total pressure. It is also not sensitive to changes in mass flow rate or inlet tube diameter [12]:

$$
D I=\frac{P_{\mathrm{max}}-P_{\mathrm{min}}}{P_{\mathrm{F}, \mathrm{AV}}} .
$$

\subsection{INLET DISTORTION CIRCUMFERENTIAL COEFFICIENT $(I D C)$}

The abbreviation for this term differs between the $I D C$ and $I D C L$ based on which source is consulted, though the $I D C$ will be used for this paper. The $I D C$ quantifies the circumferential distortion occurring at the AIP. The $I D C$ for a plane is defined as the largest value of the mean of the $I D C$ ring values of two neighbouring rings:

$$
I D C=\max \frac{I D C_{i}+I D C_{i+1}}{2} .
$$

Where the $I D C_{i}$ is defined for each ring as the difference between ring average and minimum pressure by face average total pressure:

$$
I D C_{i}=\frac{\left(P_{\mathrm{R}, \mathrm{AV}}\right)_{i}-\left(P_{\mathrm{R}, \min }\right)_{i}}{P_{\mathrm{F}, \mathrm{AV}}} .
$$

Some sources add a weighting factor to the $I D C$ calculation to capture the circumferential extent of the low total pressure on a ring $[8]$.

\subsection{INLET DISTORTION RADIAL COEFFICIENT $(I D R)$}

The radial distortion parameter is taken as the maximum value from the radial distortion of the inner (hub radial) and the outer (tip radial) ring. The definition of the ring distortion coefficient $I D R_{i}$ is exactly the same as the $R D I$ element defined previously, which can be seen clearly from the following formula. Its properties and dependencies can be inferred from the $R D I$ element. Due to the similarity, the $I D R$ is not included independently in further analysis:

$$
\begin{gathered}
I D R_{i}=\frac{P_{\mathrm{F}, \mathrm{AV}}-\left(P_{\mathrm{R}, \mathrm{AV}}\right)_{i}}{P_{\mathrm{F}, \mathrm{AV}}}, \\
I D R=\max \left(I D R_{\mathrm{in}}, I D R_{\mathrm{out}}\right) .
\end{gathered}
$$

\section{Testing Device AND METHODOLOGY}

The staff at the University of Defence developed a unique measurement device optimized for effective pressure distortion data collection of small jet engines with radial compressors. Because of the small dimensions of the inlet channel, the device was designed with just four rotating probe rakes in order to decrease the flow blockage. Instrumentation and measurement methodology is described in detail in 13; only a brief summary is presented here for the ease of reference.

\subsection{INLET DEVICE}

The engine inlet apparatus depicted in Fig. 2, consists of a short lemniscate shaped bell mouth (1), removable distortion screen (2) fixed in a collar, plastic extension tube, a rotating collar with 4 static pressure taps (3) and 4 rakes of 4 total pressure probes each (4), an engine inlet cone with struts (5), and a rear diffuser (7) for smoother air flow, all resting on a suspended platform. The four total pressure rakes are uniformly spaced around the circumference, with an inner diameter of $0.35 \mathrm{~mm}$, while the probe openings are positioned at the centres of four equal area rings. The four static pressure taps are offset by 45 degrees from each of the rakes. Additionally, there are 6 static pressure taps and 4 thermocouple sensors ( $\mathrm{K}$ type) 


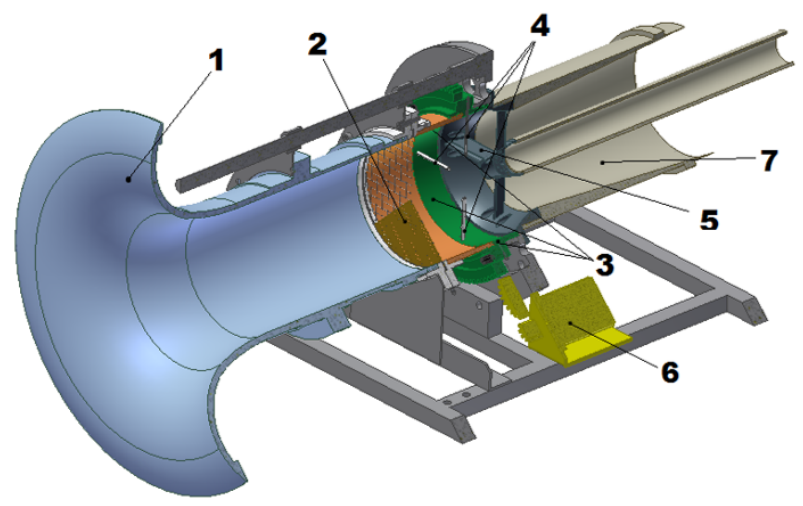

Figure 2. Inlet Section with Rotating Measurement System.
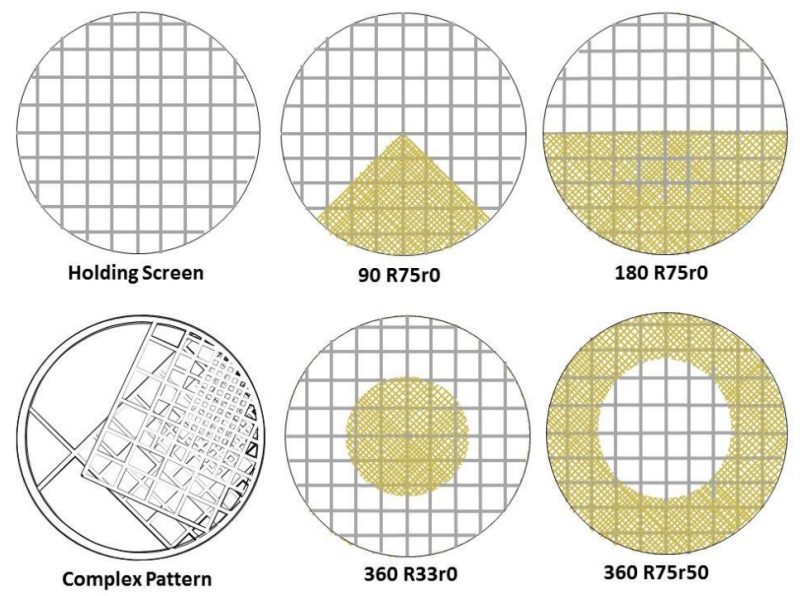

FIGURE 3. Distortion Screens.

positioned at the inlet for mass flow calculations. The collar is rotated by a stepper motor (6) and a gear system to allow for data collection at a minimum step size of $1 / 4^{\circ}$. The static and total pressure probes define the position of the AIP which is situated $34 \mathrm{~mm}$ in front of the engine inlet face. The inlet diameter is $150 \mathrm{~mm}$.

The data acquisition and rotating collar position control hardware was based on the National Instruments CompactDAQ platform. The standard [5] requirements for pressure measurement precession of $0.5 \%$ of absolute pressure was met.

\subsection{Distortion SCREENS}

The staff at the University of Defence developed a variety of distortion screens that fall into 4 categories: a holding screen, radial distortion, angular distortion, and a custom designed complex distortion. The screens and their position within the measurement device are depicted in Fig. 3.

The holding screen was intended to be used as a support scaffolding for the distortion screens under test. Various thicknesses of wire and gaps between wires were considered. The staff performed a D - Optimal Design of Experiment on different screen types and found that the screen with a wire thick-

\begin{tabular}{|c|c|}
\hline Factor Name & Levels \\
\hline & $90^{\circ}(\mathrm{R} 75 \mathrm{r} 0) ; 180^{\circ}(\mathrm{R} 75 \mathrm{r} 0)$ \\
Screen Type & $360^{\circ}(\mathrm{R} 33 \mathrm{r} 0) ; 360^{\circ}(\mathrm{R} 75 \mathrm{r} 50)$ \\
& Complex pattern \\
& Empty channel \\
\hline Screen Density & $1 \mathrm{x} ; 2 \mathrm{x} ; 3 \mathrm{x}$ \\
\hline Mass Flow Rate $[\mathrm{kg} / \mathrm{s}]$ & 1,$40 ; 1,20 ; 1,00 ; 0,70$ \\
\hline
\end{tabular}

TABLE 1. Testing factors and levels.

ness of $0.7 \mathrm{~mm}$ and a gap thickness of $9 \mathrm{~mm}$ had the smallest pressure drop and best uniformity of flow for all of the considered holding screen types. While the holding screen notably has variable size gaps on the edge of the channel, the screen was held in constant position across test runs to minimize the impact on the test results.

The angular and radial distortion screens were made from small $0.15 \mathrm{~mm}$ in diameter wires and with a gap size of $0.56 \mathrm{~mm}$ ( $60 \%$ free area). Their shapes are depicted in Fig. 3. The notation with the respective pictures describes the angular and radial extent of the screen. For example, 180 R75r0 stands for $180^{\circ}$ angular extent going from outer channel radius $(R=$ $75 \mathrm{~mm})$ to its centre $(r=0 \mathrm{~mm})$. Multiple copies of these screens were created and layered in up to 3 sheets for increased distortion intensity testing.

The complex distortion pattern is made of two screens differing by only two small support connectors, offset from each other by about $15^{\circ}$ and placed in sequence. The screens were cut from $2 \mathrm{~mm}$ thick metal sheets with 3 primarily different size square gaps. Due to overlay, the resultant size of the gaps is variable.

\subsection{Methodology}

The staff at the University of Defence developed a testing regime using Design of Experiments principals. The test consisted of 53 runs in total with the factors and their handling identified in Tab. 1. Responses presented in the following section include distortion descriptors among others. Most of the varied factor combinations were tested as a single run except for two distinct settings being repeated an extra 3 times each. Not all combinations of mass flow rate and screens were performed due to either intentional omission, or inability of the stand-alone compressor to achieve the desired mass flow rate of $1.40 \mathrm{~kg} \mathrm{~s}^{-1}$ with the most intense distortion screens types and densities.

The measurement system was run in steps of 15 degrees, that means one full run consisted of 6 angular positions to completely record readings at the AIP with a total of 96 points.

To achieve steady state measurement conditions, one step was measured for 40 seconds. Between steps, there was always a period of 5 seconds for flow stabilization after the movement of the rotating collar. The measurements were performed automatically with a check of inlet temperature and mass flow rate stability to be better than $1 \%$ within one measurement. The 


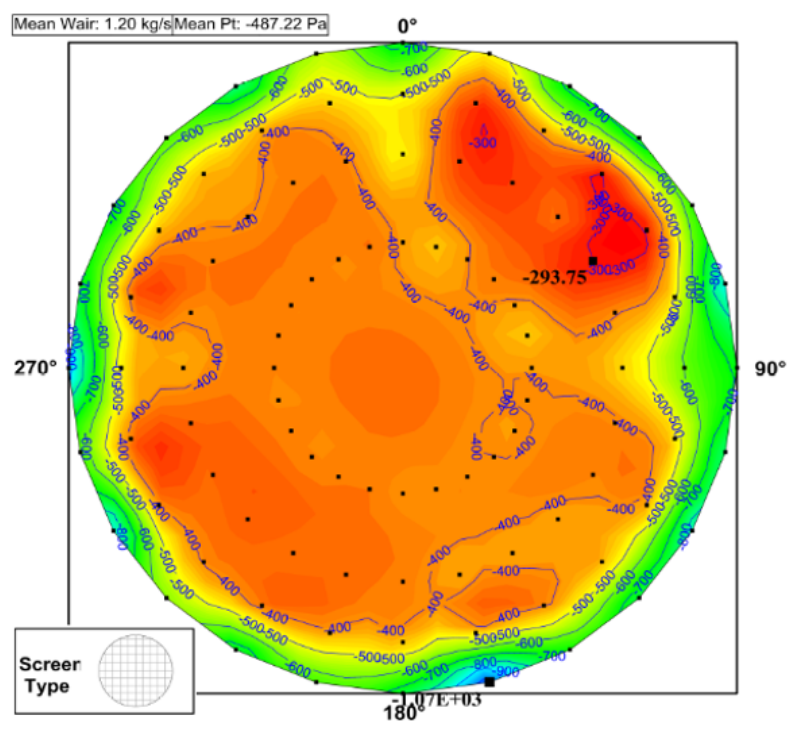

FiguRE 4. Holding screen distortion pattern.

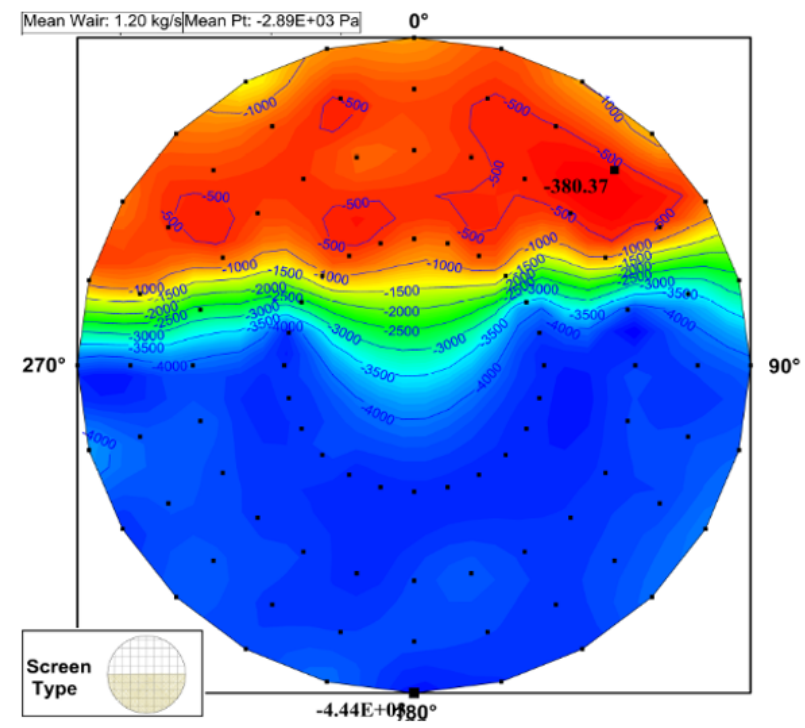

Figure 5. Angular distortion pattern (2x180 R75r0).

length of the recording represents the best trade-off between the overall recording length and accuracy of results and met the recommendations of published standards and other sources [5, 9, 14.

The sampling rate for the pressure measurements was $1000 \mathrm{~Hz}$. Measured values were averaged, corrected to the ISA, and then used for distortion descriptor calculations and saved. Saved data from separate measurements were processed into report sheets, like the one in Fig. 4. Calculated descriptors were analysed in order to evaluate dependencies on screen shape, density, and air mass flow rate.

\section{Results}

The results presented here serve to highlight the key findings of the test, although they represent only a partial exhibition of the full data set. Raw data and more detailed methodology is presented in [15] and available on request from the authors.

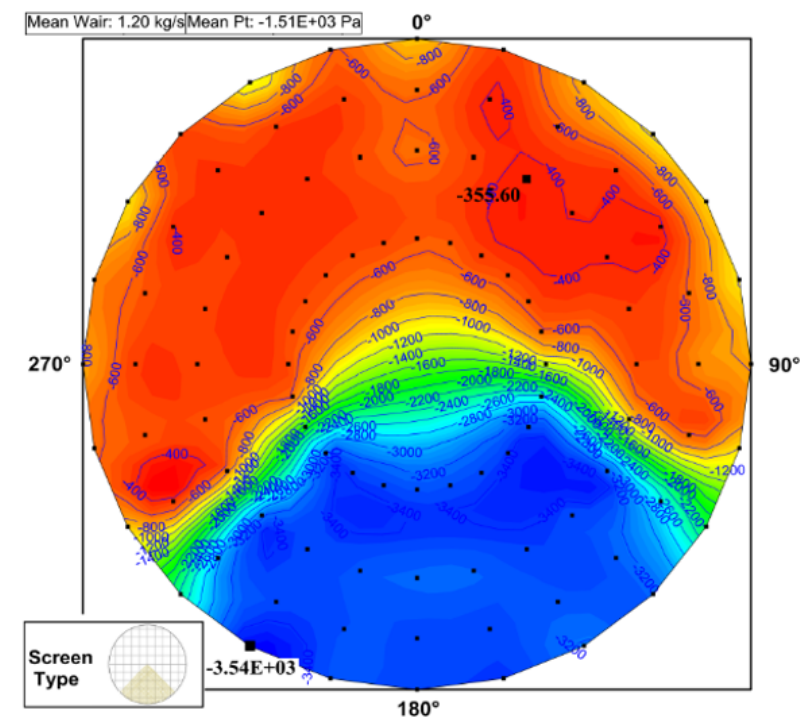

Figure 6. Angular Distortion pattern (2x90 R75r0).

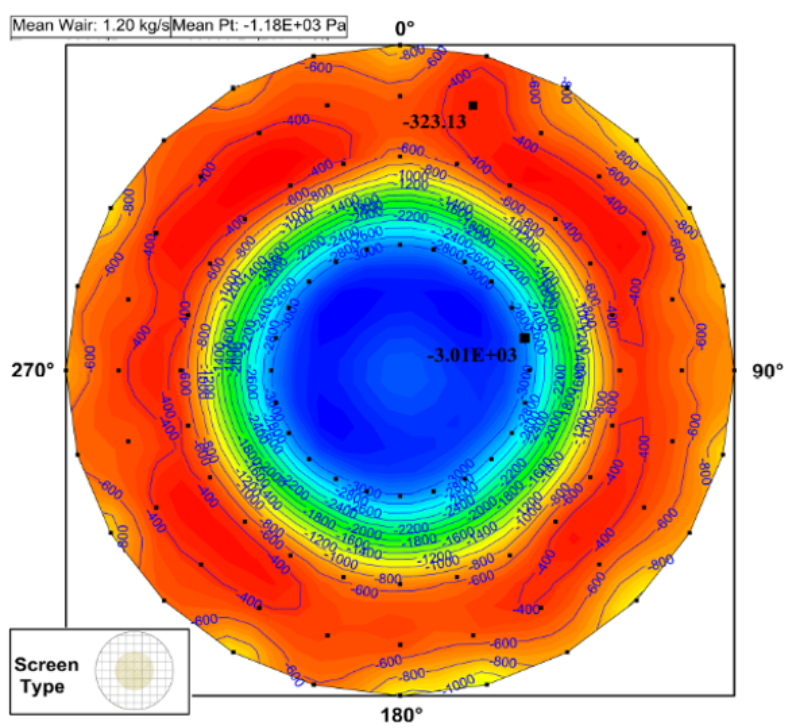

FiguRE 7. Radial distortion pattern (2x360 R33r0).

This section begins with an overview of the presented data and continues with the analysis of the data broken down by the distortion descriptor in a matching order to that found in Section 2 . Figures 4 9 are key exemplars from the collected data set and depict the primary report image the authors used for evaluating the response of the distortion descriptors to different distortion screens. The values in the colour maps are total pressure drops relative to the atmospheric pressure.

It is possible to see that with the complex pattern screen (Fig. 9), which does not require the holding screen, the values in unblocked areas goes up to $0 \mathrm{~Pa}$ pressure drop. For screens that incorporate the holding screen, the pressure drop on the holding screen ranges from about 400 to $500 \mathrm{~Pa}$.

Figures 11, 12, 13 , and 14 show the change of individual descriptor values (y axis) with the mass flow rate ( $\mathrm{x}$ axis). The primary columns are for different 


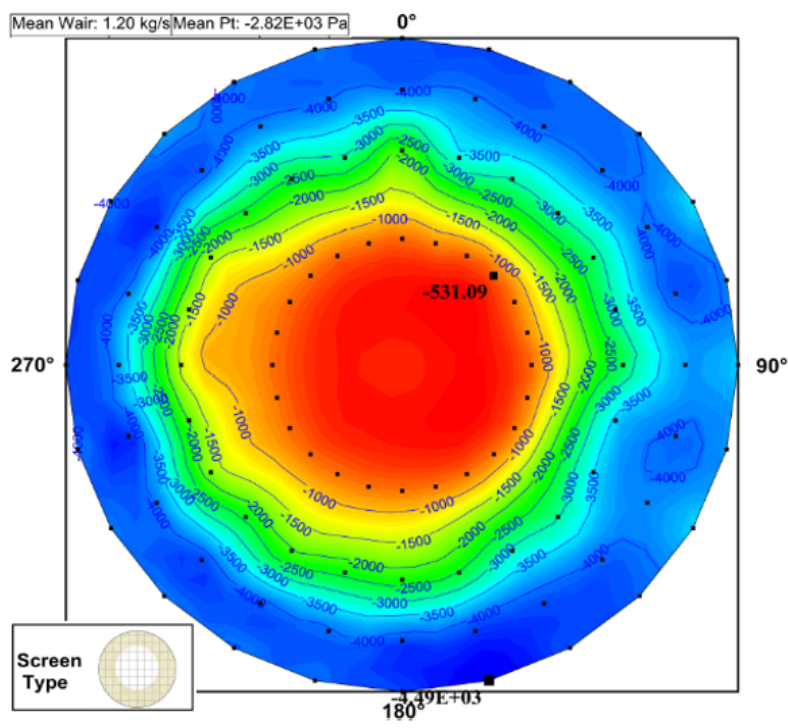

Figure 8. Radial distortion pattern (2x360 R75r50).

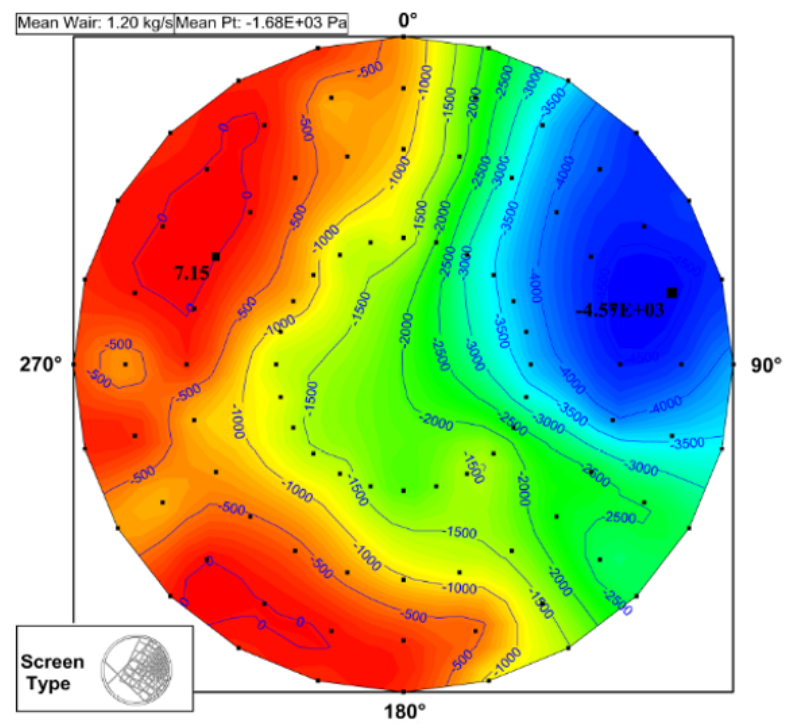

Figure 9. Complex distortion pattern.

screen shapes and densities (number of mesh layers). The complex pattern is added in all the figures for the sake of comparison. In Fig. 15 the influence of the screen density on the relative change of the descriptor is depicted. The behaviour of the descriptors is different for radial and circumferential distortions, thus the results are also separated in the figures and respective text analysis.

\subsection{SAE DESCRIPTORS}

The Circumferential Distortion Intensity ( $C D I)$ element measurements of separate rings were very similar as was their rate of change with regards to mass flow rate, screen shape, and density. As a result, unless stated otherwise, their properties described are true for all of the rings. According to the definition, the $C D I$ should only be sensitive to angular patterns. However for the tip radial pattern 2x360 R75r50 (Fig. 11 bottom and Fig. 8) it

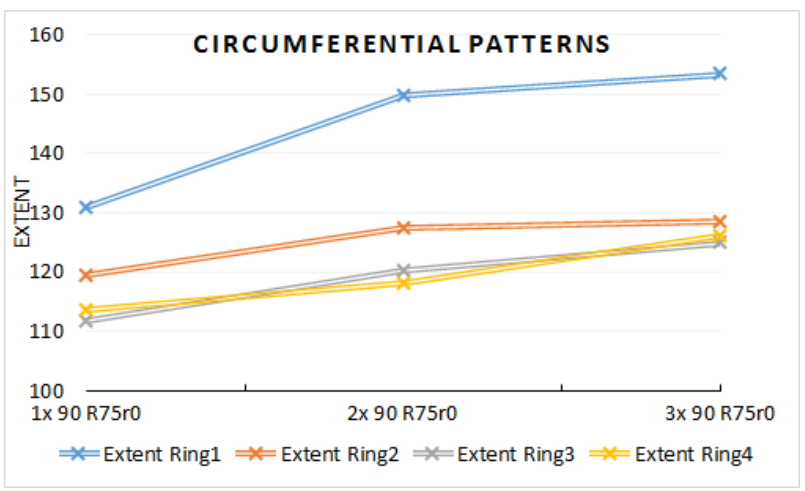

Figure 10. Extent dependency on screen density for 90 R75r0 screen.

rises up to $5 \cdot 10^{-3}$ for 1 and $1.2 \mathrm{~kg} / \mathrm{s}$ (for the second ring). That is the same value as with the angular pattern $1 \times 180 \mathrm{R} 75 \mathrm{r} 0$ at $1 \mathrm{~kg} / \mathrm{s}$ or other angular patterns at $0.7 \mathrm{~kg} / \mathrm{s}$ (Fig. 11). This is due to a fact that the ring 2 is in a region of significant radial pressure gradient, which is not totally axisymmetric with the ring. That is causing significant radial distortion pattern at ring 2, where the pressure level in the radial direction is ranging by more than circa $50 \%$ of the average ring pressure. When only the holding screen is employed, the $C D I$ values are approximately $4 \cdot 10^{-4}$ (Fig. 4) and rise up to $2 \cdot 10^{-2}$ for the double density $90^{\circ}$ angular pattern. That is an overall change of almost two orders of magnitude. The $C D I$ also varies significantly with the level of mass flow rate. When mass flow rate doubles, the intensity increases approximately 4.5 times for both radial and circumferential screen patterns. For circumferential distortions, the intensity grows by approximately $50 \%$ when doubling the screen density (Fig. 15). As long as the values of the $C D I$ for radial screens can rise up to the level of values for the angular patterns it is important to observe its change with radial screen patterns density, which was rather inconsistent. The intensity rose when the second screen was added and dropped with the third screen addition (Fig. 15 bottom). It is possible that this effect is caused by turbulences at the edge of the screen, although these behaviours would have to be further evaluated.

An important fact the team discovered is that the $C D I$ for rings 1 to 3 was the only circumferential descriptor that gave higher values for the $3 \mathrm{x} 90 \mathrm{R} 75 \mathrm{r} 0$ screen than for the complex pattern (Tab. 2). In other words, according to the $C D I$ the triple density $90^{\circ}$ screen creates a more intense distortion than the complex pattern, but all other descriptors suggest the reverse. This is the only pair of distortion patterns where different descriptors showed different results.

The Radia Distortionl Intensity $(R D I)$ element, defined as relative difference to the face average pressure, can be either negative or positive. Its absolute 


\begin{tabular}{|c|c|c|c|c|c|c|c|}
\hline & CDI Ring1 & CDI Ring2 & CDI Ring3 & CDI Ring4 & IDC & DC60 & DI \\
\hline 3x 90 R75r0 & 0,018 & 0,02 & 0,021 & 0,017 & 0,023 & $-1,058$ & 0,036 \\
\hline Complex screen & 0,013 & 0,018 & 0,019 & 0,019 & 0,03 & $-1,26$ & 0,046 \\
\hline
\end{tabular}

TABLE 2. 3x90 R75r0 and Complex screen comparison for $1.2 \mathrm{~kg} / \mathrm{s}$ mass flow rate.

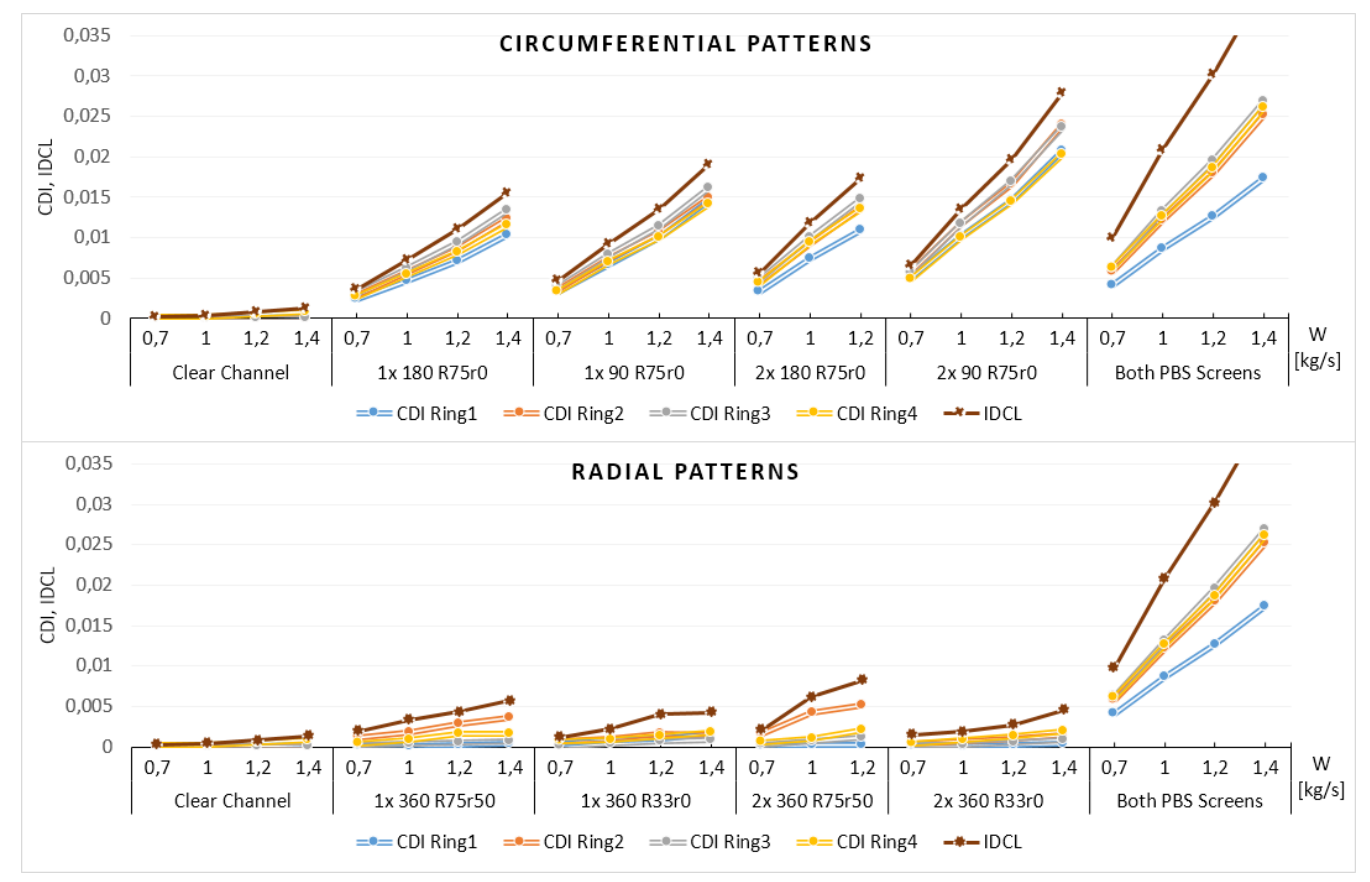

Figure 11. Circumferential intensity change with mass flow rate.

value ranged from $10^{-3}$ for the holding screen with circumferential patterns to $2 \cdot 10^{-2}$ for radial distortion patterns. There were significant differences of the absolute values between the most inner and outer ring for radial patterns. Specifically, for the hub radial pattern (2x360 R33r0), an up to eightfold difference in the absolute values could be observed (Fig. 12). The rise with the mass flow rate is comparable to the $C D I$ element and it behaves similarly in regards to screen density changes as well. For radial patterns, the $R D I$ element increases by approximately $50 \%$ when doubling the screen density. With circumferential patterns, the $R D I$ absolute value change was inconsistent; for some rings it rose, for some it dropped (Fig. 15). This different rate of change is not considered to be disturbing due to the small absolute values of the $R D I$ elements with angular screens.

The Extent element of the SAE distortion descriptor was about $20^{\circ}$ to $40^{\circ}$ larger than the size of the circumferential distortion screen employed and highest for the most inner ring (Figures 5 and 6). It remained unchanged with changing mass flow rate, but it changed with varying screen density (Fig. 10).

The Multiple per revolution $(M P R)$ element was exactly 1 for the angular distortion patterns. For radial patterns, it ranged between 1 and 2 . Nevertheless, owing to respective circumferential intensities elements, the extent and MPR are quite unimportant for radial patterns.

\subsection{Distortion Coefficient (DC60)}

The $D C 60$ (90) values are always negative and have higher absolute values when the distortion pressure drops is greater. Due to the extent of the circumferential patterns compared $\left(90^{\circ}, 180^{\circ}\right.$ and radial $)$, the values of the $D C 60$ and $D C 90$ were the same except for the complex pattern. Their values were about -0.02 for both the holding screen and radial patterns (i.e., undistorted flow; Fig. 13). They changed from -0.45 up to -1.3 for the angular and complex pattern respectively. The descriptor is only slightly dependent on mass flow rate. Over the measured range of 0.7 to $1.4 \mathrm{~kg} / \mathrm{s}$, it changed by about $2 \%$ with the minimal absolute value usually appearing at $1 \mathrm{~kg} / \mathrm{s}$. The changes due to screen density are similar to those for the SAE CDI element, which are approximately $50 \%$ when doubling the screen density (Fig. 15).

\subsection{Distortion index $(D I)$}

The $D I$ is the only descriptor that is sensitive to both circumferential and radial distortions. It has a strong dependency on the mass flow rate. When the flow rate doubles, the $D I$ rises more than three times (Fig. 14). Dependency on the screen density is similar to the other descriptors, which is, again, approximately $50 \%$ when doubling the screen density (Fig,15). 


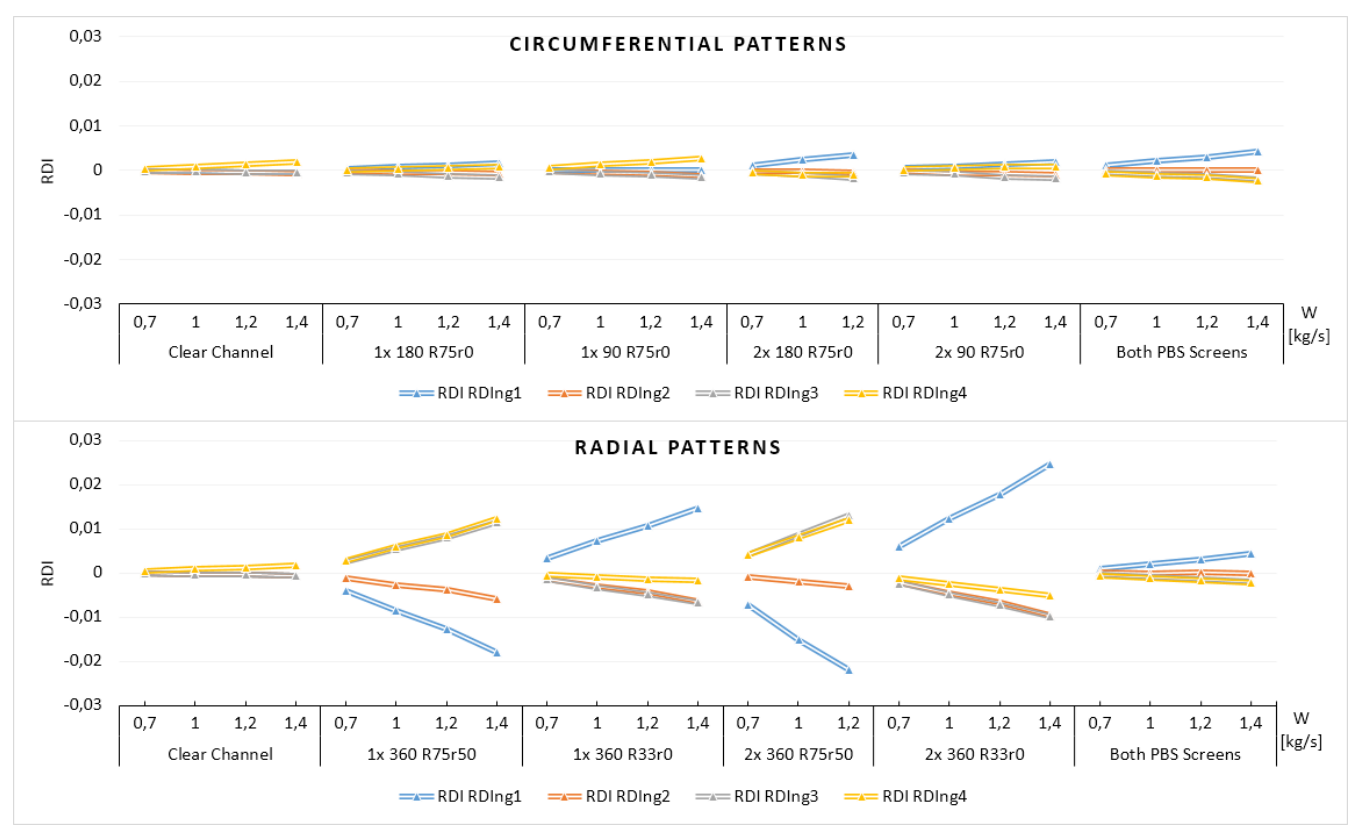

FiguRE 12. Radial intensity change with mass flow rate.

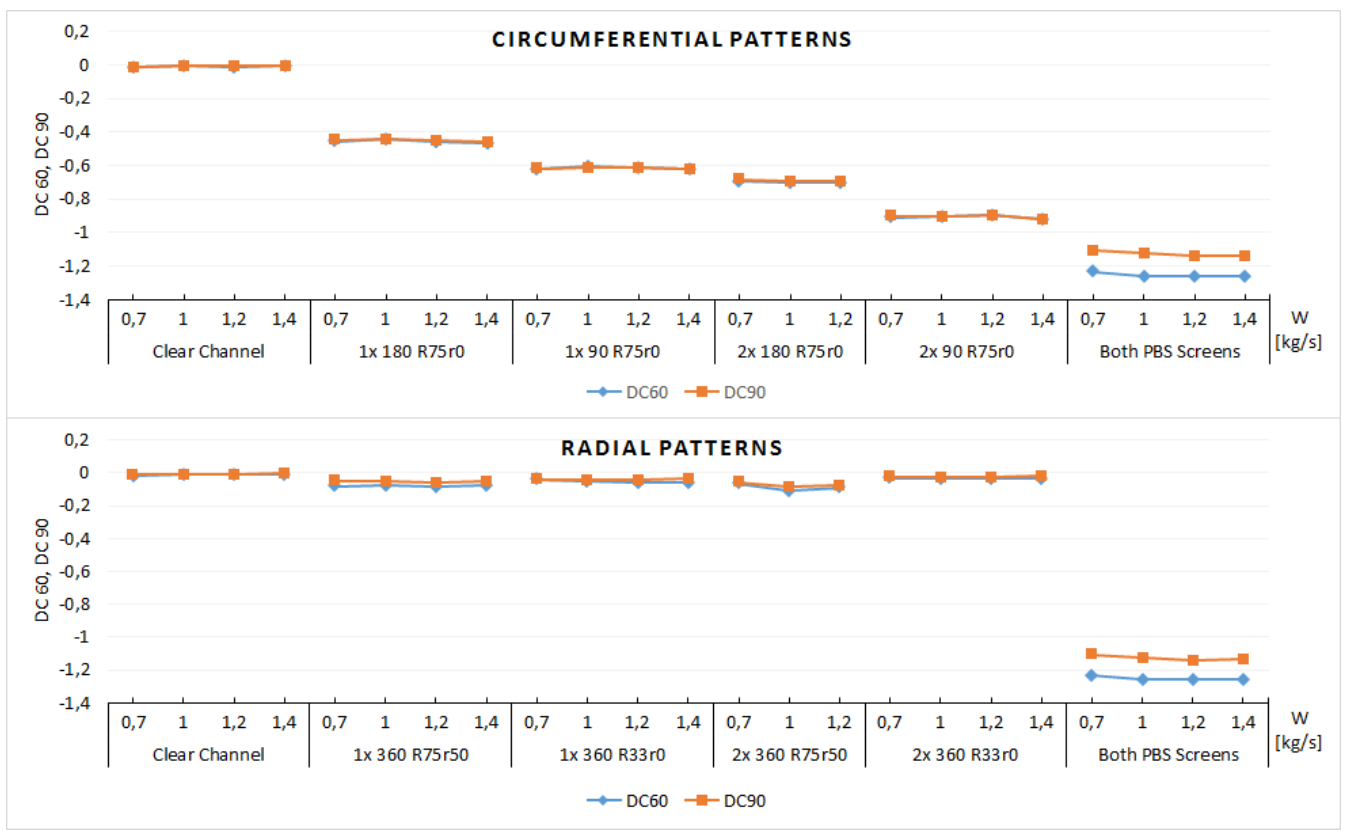

Figure 13. DC60 (90) change with mass flow rate.

\subsection{INLET DistORTION CIRCUMFERENTIAL COEFFICIENT $(I D C)$}

The $I D C$ coefficient behaved very similarly to the $C D I$ element. It has only slightly higher values ranging from $3 \cdot 10^{-3}$ to $3 \cdot 10^{-2}$. The $I D C$ change with the air mass flow rate and screen density was the same as the one for the CDI (Figures 11 and 15).

\section{Conclusions}

Staff at the University of Defence completed more than fifty runs on a jet engine inlet model device with specially designed total pressure distortion patterns. The goal was to test and compare commonly used descriptors in order to find some that would describe the distortion with sufficient fidelity and reasonable complexity. The selected descriptor will be used in further research with the TJ100 small jet engine, mainly for surge margin analysis.

The least suitable descriptor seems to be the distortion index $(D I)$, since it is sensitive to both radial and angular distortion and it is also very sensitive to air mass flow rate. Thus its value gives little to no information about the distortion size or intensity. The same $D I$ value can be obtained for an intense radial distortion as for a low intensity angular one. It would be very problematic to correlate it with the surge margin change.

The $I D C$ and $I D R$ descriptors behave very similarly 


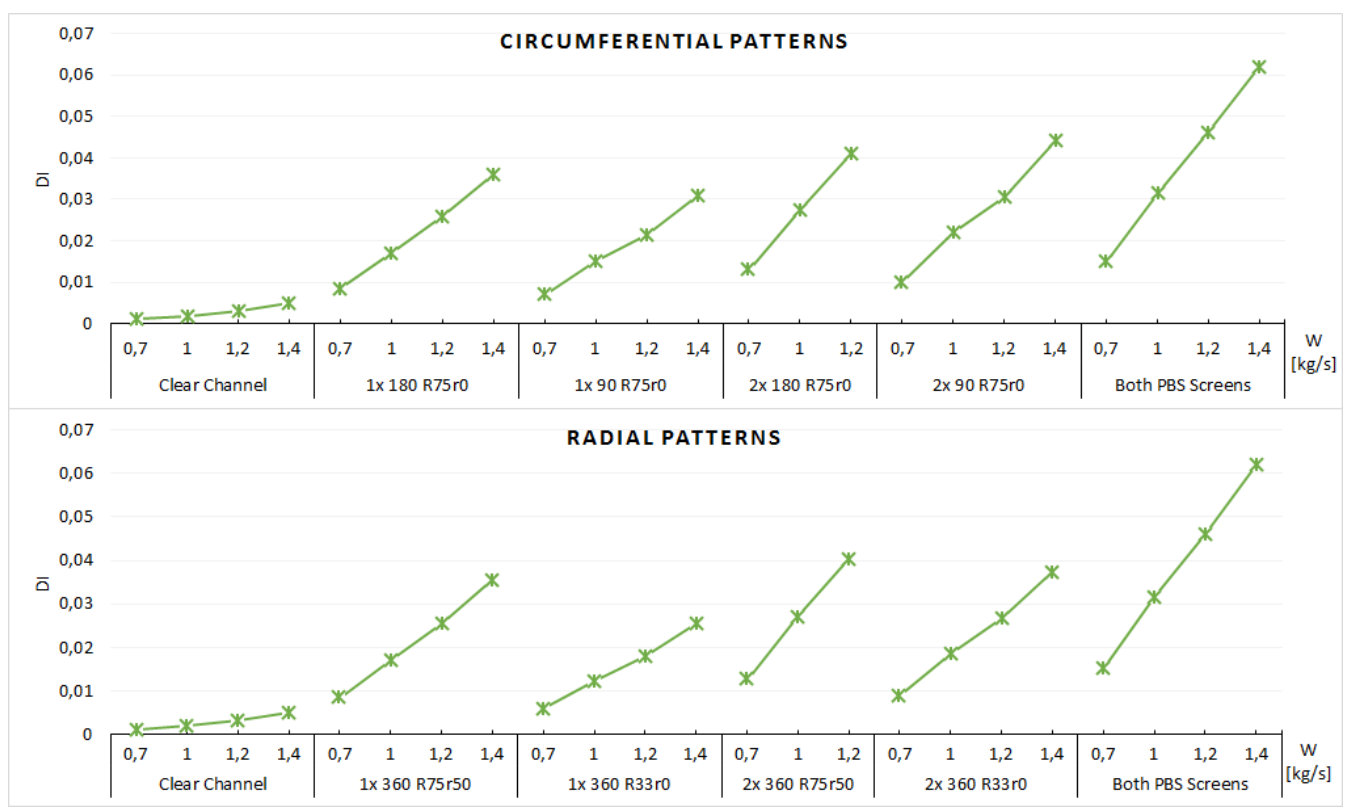

FIGURE 14. Distortion Index change with mass flow rate.

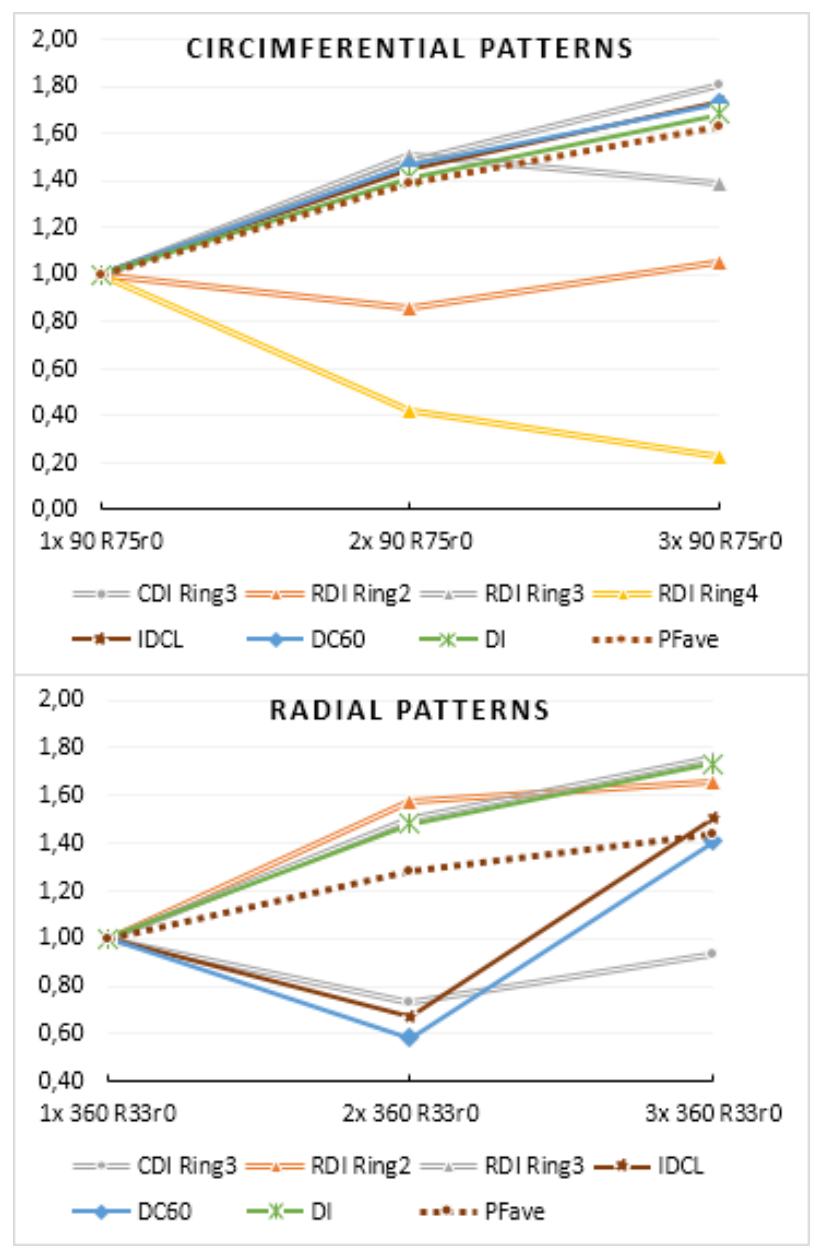

Figure 15. Radial and angular patterns relative change with screen density. to the $C D I$ and $R D I$ elements of the SAE descriptor and their change with the mass flow rate, screen shape, and density are almost the same. Thus, unless there is a proof of significantly different sensitivity of the compressor surge margin to the distortion for different rings, it seems a justifiable simplification to use the $I D C$ and the IDR instead of the SAE $C D I$ and $R D I$ elements or radially average the SAE ring coefficients. An important feature of both the $I D C$, the $I D R$ and the SAE descriptors is the significant change with the mass flow rate. The intensity values can be the same for a single screen as for triple density screens if the mass flow rate is lowered by $30 \%$ for the triple density screen. It is also clear that the circumferential and radial sensitivities need to be evaluated separately for different mass flow rates.

The DC60 (90) coefficient is almost independent of the mass flow rate which is probably due to its reliance on the inlet dynamic pressure $\left(\mathrm{P}_{\mathrm{DI}}\right)$, i.e., the flow velocity. Because of its constant value across mass flow rates, it is possible to use this coefficient for simple comparison of the distortion significance between different references without knowing the related flow velocities. For example in [10], it is stated that the most intense values of the $D C 60$ in the operational envelope are -0.2 for a civil subsonic transport and -0.9 for a military fighter aircraft. These values can be directly compared with tested distortions. The main disadvantage of the $D C 60$ (90) is that it is designed to be used only for circumferential distortion patterns and lacks a counterpart for the radial patterns.

When focusing on evaluating the circumferential distortion pattern in particular, the DC60 (90) coefficient stands out due to its simplicity and mass flow rate independence. As a result, the DC60 coefficient was chosen as the primary descriptor for further tests 
with the TJ100 engine at the University of Defence. If the compressor sensitivity to radial distortion patterns proves to be more than negligible, the set of SAE descriptors needs to be used.

One important discovery arose from comparing the descriptors. Specifically, the fact that the SAE descriptor behaved differently versus others, when comparing the distortion of the $3 \times 90$ R75r0 screen with the complex pattern, although the trend with changing screen density was the same, is noteworthy. This means that based on the descriptor considered, there is a different direction in the trend not just different rate of change. However, the real surge margin can either rise or fall and thus will comply with the $C D I$ or the rest of the descriptors. Further research will be conducted to evaluate this in depth and to find what is the real surge margin change in these two particular cases.

\section{LIST OF SYMBOLS}

$A I P$ Aerodynamic interface plane

$(C D I)$ Circumferential distortion intensity elem. [-]

$C_{i} \quad$ Constant (offset) term for ring i $[-]$

DC60 Distortion coefficient of 60 degrees $\left[^{\circ}\right]$

$D I$ Distortion index [-]

$I D C(L)$ Inlet distortion circumferential coefficient [-]

$I D R$ Inlet distortion radial coefficient [-]

$I S A$ International standard atmosphere

$K C_{i}$ Circumferential distortion sensitivity for ring i [-]

$K R_{i} \quad$ Radial distortion sensitivity for ring i [-]

$M P R$ Multiple per revolution

$N$ Number of instrumentation rings $[-]$

$P$ Pressure, Total pressure $[\mathrm{Pa}]$

$R$ Outer radius $[-]$

$r$ Inner radius $[-]$

$(R D I)$ Radial distortion intensity element [-]

$S A E$ Society of Automotive Engineers

$W$ Mass flow rate $[\mathrm{kg} / \mathrm{s}]$

$(M P R)$ Circumferential distortion element [-]

$\left(P_{\mathrm{R}, \mathrm{AV}}\right)_{i} \quad$ Ring $i$ average total pressure $[\mathrm{Pa}]$

$\left(P_{\mathrm{L}, \mathrm{AV}}\right)_{i}$ Average total pressure of lower pressure region of ring $i[\mathrm{~Pa}]$

$\theta^{-}$Circumferential distortion extent element $[-]$

$60 / 90$ Size of low pressure region in degrees

\section{SUBSCRIPTS}

AV Average

DI Dynamic Inlet

F Face

$i \quad$ Number of ring of total pressure probe

L Low pressure region

Q Number of lower pressure regions

R Ring

\section{ACKNOWLEDGEMENTS}

This work was funded by the Ministry of Defence of the Czech Republic under the framework of Project for Workplace Development — DZRO K205.

\section{REFERENCES}

[1] R. Rademakers. Approach for an Optimized Evaluation of Pressure and Swirl Distortion in S-Shaped Engine Inlet Configurations. Propulsion and Energy Forum. American Institute of Aeronautics and Astronautics, 2014. DOI:10.2514/6.2014-3594.

[2] J. Seddon, E. Goldsmith. Intake Aerodynamics. AIAA Education Series. AIAA, Reston, VA. DOI:10.2514/4.473616

[3] J. Longley, E. Greitzer. Inlet Distortion Effects in Aircraft Propulsion System Integration, book section 6, p. 18. AGARD, 1992. ISBN: 92-835-0674-X.

[4] W. Cousins. History, philosophy, physics, and future directions of aircraft propulsion system/inlet integration. In ASME Turbo Expo 2004, p. 16. ASME. DOI:10.1115/GT2004-54210

[5] SAE. Standard AIR 1419B, Inlet Total-Pressure Distortion Considerations for Gas Turbine Engines, Revised May 2013.

[6] A. Campbell. An Investigation of Distortion Indices for Prediction of Stalling Behavior in Aircraft Turbine Engines. Thesis, 1981.

[7] R. Hercock, D. Williams. Aerodynamic response. Agard LS 72, Distortion Induced Engine Instability p. 41, 1974.

[8] C. Bissinger, Norbet, T. Breuer. Basic Principles Gas Turbine Compatibility - Intake Aerodynamic Aspects, vol. 8, book section EAE487. John Wiley and Sons, 2010. DOI:10.1002/9780470686652.eae487

[9] SAE. Standard ARP 1420B, Gas Turbine Inlet Flow Distortion Guidelines, Revised February 2002.

[10] P. Walsh, P. Fletcher. Gas Turbine Performance, Second Edition. Blackwell Science, Limited, 2004. DOI:10.1002/9780470774533

[11] J. Kurzke. Effects of inlet flow distortion on the performance of aircraft gas turbine. In Proceedings of the ASME Turbo Expo 2006. DOI:10.1115/GT2006-90419.

[12] M. Sivapragasam. Numerical and experimental investigation on flow distortion in an aero engine air intake. SASTECH Journal, MSRSAS 6:28-35, 2007.

[13] J. Pecinka. Small engine inlet distortion testing device. In ICMT 2015, p. 6. DOI:10.1109/MILTECHS.2015.7153691

[14] AGARD. AR-270 Air Intakes for High Speed Vehicles. Advisory Report. AGARD, NEUILLY SUR SEINE, 1991. ISBN: 92-835-0637-5.

[15] J. Pecinka. TJ100 distortion screen test report. Report, University of Defence, 2014. 